\title{
Identification of larval fish in mangrove areas of Peninsular Malaysia using morphology and DNA barcoding methods
}

\begin{abstract}
The identification of larval fish has been an important morphological issue in marine biology due to the dramatic transformations that most species undergo from early larval stages to adulthood. Insufficient morphological diagnostic characters in larval fishes made it easy to misidentify them and a difficult process to key to genus and species level. The experiment aims to find out, by applying DNA barcoding, how consistent the morphological identifications can be among larval fish. Larval fish were mainly collected using plankton nets around mangrove areas in Pendas (Johor), Setiu (Terengganu), Pekan (Pahang) and Matang (Perak) Malaysia between April 2015 and October 2015. A total of 354 samples were morphologically identified, mostly to the family level and a few to the genus level. Larval fish ranged from $1.5 \mathrm{~mm}$ to $31 \mathrm{~mm}$ of total length, with the most abundant individuals being $<3 \mathrm{~mm}$. Among them, a total of 177 individuals were selected for DNA barcoding analyses. Molecular works involved polymerase chain reaction (PCR) and sequencing of mitochondrial Cytochrome c Oxidase I (COI) gene fragment (655 base pairs) methods. DNA barcoding enabled all samples to be identified down to species level. The overall genetic identities ranged from $91 \%$ to $100 \%$. Morphological identification classified the specimens into 19 families and 11 genera while DNA barcoding identified them into 19 families 33 genera and 40 species. A comparison between the two methods showed a mismatched identification of $42.6 \%$ where the accuracy percentage for morphological identification was moderate for the family level (67.8\%) but was low for genus level identification (30\%). The DNA barcoding method also managed to successfully identify $86.4 \%$ of the samples up to their species level where morphological method has failed to do so. The most misidentified families in the study were Blenniidae, Sparidae, Apogonidae Ambassidae and Monachantidae while almost all samples from the family Gobiidae and Engraulidae were correctly identified to family level because of their distinct morphology. In conclusion, taxonomic studies of larval fish should continue using combination of both morphology and DNA barcoding methods. Morphological identification should be more conservative i.e., when in doubt, it is better to key only to family and not to the genus and species level. DNA barcoding is a better method for deeper taxonomic levels identification with the existence of robust sequence reference libraries and should be able to validate the accuracy of traditional larval fish identification.
\end{abstract}

Keyword: Larval fish; Mangrove areas; Peninsular Malaysia; Morphology; Morphological identification; DNA barcoding; DNA barcoding method 